\title{
Hybrid Adaptive Filter development for the minimisation of transient fluctuations superimposed on electrotelluric field recordings mainly by magnetic storms
}

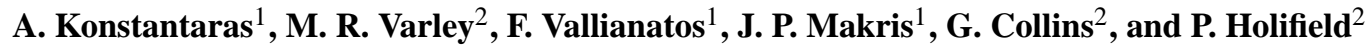 \\ ${ }^{1}$ Technological Educational Institute of Crete, Chania, Crete, 731 33, Greece \\ ${ }^{2}$ ADSIP Research Centre, Department of Technology, University of Central Lancashire, Preston, PR1 2HE, UK
}

Received: 17 July 2006 - Revised: 23 October 2006 - Accepted: 23 October 2006 - Published: 30 October 2006

\begin{abstract}
The method of Hybrid Adaptive Filtering (HAF) aims to recover the recorded electric field signals from anomalies of magnetotelluric origin induced mainly by magnetic storms. An adaptive filter incorporating neuro-fuzzy technology has been developed to remove any significant distortions from the equivalent magnetic field signal, as retrieved from the original electric field signal by reversing the magnetotelluric method. Testing with further unseen data verifies the reliability of the model and demonstrates the effectiveness of the HAF method.
\end{abstract}

\section{Introduction}

Prior to an earthquake, there is energy storage in the seismogenic area, the release of which results in a number of micro-cracks, which in effect produce a weak electric signal. Initially, there is a rapid rise in the number of propagating cracks, which creates a transient electric field. The whole process lasts in the order of several tens of minutes, and the resulting electric signal is considered as an electric earthquake precursor (EEP) (Tzanis and Vallianatos, 2001; Varotsos and Alexopoulos, 1984). EEPs appear on recordings of the Earth's electric field $(E)$ and their recognition is mainly prevented by the probable appearance of severe transient fluctuations induced mainly by magnetic storms (or sub-storms), and/or other physical and anthropogenic types of noise (Varotsos and Alexopoulos, 1984). At the occurrence of a magnetic storm (Varotsos and Alexopoulos, 1984; Vallianatos et al., 2002) at an observation point, which usually lasts for several tens of minutes, significant disturbances are recorded upon the Earth's magnetic field measurements $(H)$. This has an immediate effect on the electric field (Cagniard, 1953; Kaufman and Keller, 1981), resulting in a

Correspondence to: A. Konstantaras

(akonstantaras@chania.teicrete.gr) different spread of the latter. When an EEP occurs during significant magnetic fluctuations the disturbances can be so severe that its recognition may not be possible.

The recovery of EEP signals has long puzzled the scientific community but very few approaches have shown promising results (Hadjioannou and Vallianatos, 1993; Rovithakis and Vallianatos, 2000; Konstantaras et al., 2006b). The method of "the subtraction of the telluric inductive component from electric field recordings" (Hadjioannou and Vallianatos, 1993) developed in the early 1990s has the disadvantage of the assumption that the existence of preseismic magnetic fields does not influence the estimated induced electrical components. In an effort to ignore the impedance tensor, a method incorporating neural networks has been developed (Rovithakis and Vallianatos, 2000), where the neural network is trained to predict the Earth's electric field. Thus, any electrotelluric anomaly due to a seismoelectric source is enhanced on the prediction error signal, measured as the difference of the recorded and the predicted electric field signal, as it is an "external" signal added on and not a genuine part of the electric field. However the effectiveness of the method relies on the assumption that at the time of their occurrence EEPs are not accompanied by any significant magnetotelluric anomalies.

This paper presents the Hybrid Adaptive Filtering method, a two-step process incorporating neuro-fuzzy technology for the development of adaptive filters trained to minimize the effect of magnetotelluric disturbances presented upon the electric field.

\section{Hybrid Adaptive Filtering}

The HAF method is a two-step process (Fig. 1) based upon the magnetotelluric method (Kaufman and Keller, 1981), which specifies that at an observation point the relation between the electric and magnetic field at a particular frequency

Published by Copernicus GmbH on behalf of the European Geosciences Union. 


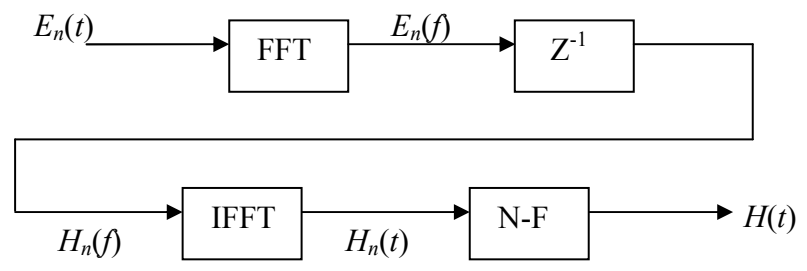

Step 1: Hybrid Adaptive Filtering

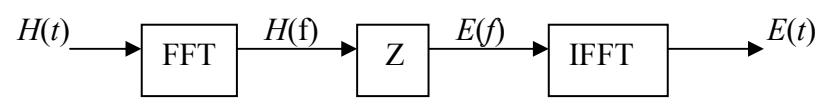

Step 2: Reconstruction

Fig. 1. Flow chart analysis of the two-step operation of the HAF method.

is linear, thus the magnetotelluric impedance tensor $Z$ is defined in matrix notation as: $\underline{E}=Z \underline{H}$. During the first step the noise-affected recorded electric field signal $E_{n}$ is transformed to the frequency domain $E_{n}(\omega)$ and the equivalent embedded-in-noise magnetic field signal $H_{n}(\omega)$ is calculated by reversing the magnetotelluric method, e.g. $\underline{H}=Z^{-1} \underline{E}$. The noise embedded magnetic field signal is then transformed from the frequency domain to the time domain $H_{n}$.

In order to minimize any probable external severe fluctuations from the recovered magnetic field signal $H_{n}$, a neurofuzzy model is incorporated, trained to operate as an adaptive filter. To reliably assess its performance, the neuro-fuzzy model is trained with simulated magnetic field signals featuring the main characteristics of the Earth's magnetic field, i.e. chaotic time-series with a spectral density function that satisfies the relationship $S(\omega) \propto \omega^{-a}$. The neuro-fuzzy model is trained by a hybrid algorithm (Jang, 1993) to identify the signal's main characteristics and reproduce the current input sample $n$ based upon previous samples, i.e. $n-1, n-2$, $n-3$. In the event of external fluctuations, such as magnetic storms, the neuro-fuzzy model compresses the amplitude of the input sample $n$ to the expected level of the unaffected magnetic field signal. Thus, the resulting magnetic field signal $H$ has its external distortions minimized to a satisfactory level, no greater than that of random noise appearing on the recorded data estimated at approximately $20 \mathrm{~dB}$ (Makris, 1997; d'Erceville and Kunetz; Groom, 1988).

The second step is the reconstruction process, which computes the electric field signal based upon the "cured" magnetic field signal $H$. As a result, any external distortions of magnetic origin are minimized upon the reconstructed electric field signal to a satisfactory level, which approximates the level of standard random noise appearing on the recorded data, i.e. $20 \mathrm{~dB}$ (Makris, 1997; d'Erceville and Kunetz; Groom, 1988). According to the magnetotelluric method, the "cured" magnetic field signal $H$ is transformed to the frequency domain $H(\omega)$ for the equivalent electric field signal $E(\omega)$ to be computed throughout the relevant frequency spectrum, i.e. $E(\omega)=Z(\omega) H(\omega)$. The resulting signal $E(\omega)$ is finally transformed to the time domain providing the reconstructed electric field signal $E$ with minimal external disturbances.

\section{Neuro-Fuzzy model architecture and training}

Neuro-Fuzzy models are neural networks with intrinsic fuzzy logic abilities (Konstantaras et al., 2002), i.e. the weights of the neurons in the network define the premise and consequent parameters of a fuzzy inference system. Premise parameters determine the shape and size of the input membership functions, whilst consequent parameters determine the characteristics of the output membership functions and define the rules guiding the fuzzy inference system. To generate an initial fuzzy inference system, i.e. to produce an initial set of premise and consequent parameters, subtractive clustering (Jang et al., 1997) is applied on the input data of an input/output data set. The structure of the neuro-fuzzy model depends on the number of inputs and input membership functions per input. Each rule and membership function is represented by a single neuron. The number of rules guiding the fuzzy inference system is equal to the number of membership functions per input to the power of the total number of inputs of the system. A single neuron is used as bias to define the importance of either rule in the system. Each rule is associated to a single output membership function. A single neuron is used to produce a crisp output (defuzzification). The current and three previous samples of the recorded magnetic field signal $H_{n}$ are used as inputs (layer 1) to the neuro-fuzzy model (Fig. 2) introducing recursion to the network (input data set). The required output is the magnetic field signal with minimal additional disturbances (output data set).

The layer by layer operation of the neuro-fuzzy model is described in this paragraph: In layer 1, the present and three previous samples of the recorded signal are used as inputs ( $A$ to $D$ ) to the network. In layer 2 , every node $i$ in this layer is an adaptive node with a node function: $O_{1, i}=\mu A_{i}(x)$, for $i=1,2$, or $O_{1, i}=\mu B_{i-2}(y)$, for $i=3,4$, or $O_{1, i}=\mu C_{i-4}(z)$, for $i=5,6$, or $O_{1, i}=\mu D_{i-6}(k)$, for $i=7,8$, where $x$ (or $y$, or $z$, or $k$ ) is the input to node $i$ and $A_{i}$ (or $B_{i}$, or $C_{i}$, or $D_{i}$ ) is the equivalent membership function. The type of MFs $A, B, C$ and $D$ is that of the generalized bell function: $\mu A_{i}(x)=1 /\left(1+\left|\frac{x-c_{i}}{a_{i}}\right|^{2 b}\right)$, where $\left\{a_{i}, b_{i}, c_{i}\right\}$ are the premise parameters of the network (Konstantaras et al., 2004) which determine the shape and size of the MF. In layer 3 , every node in this layer is a fixed node calculating the normalized firing strength of either rule: $O_{2, i}=\overline{w_{i}}=\frac{w_{i}}{\sum_{i} w_{i}}$, where $w_{i}=\mu A_{i}(x) \mu B_{i}(y) \mu C_{i}(z) \mu D_{i}(k)$. In layer 4 , every 


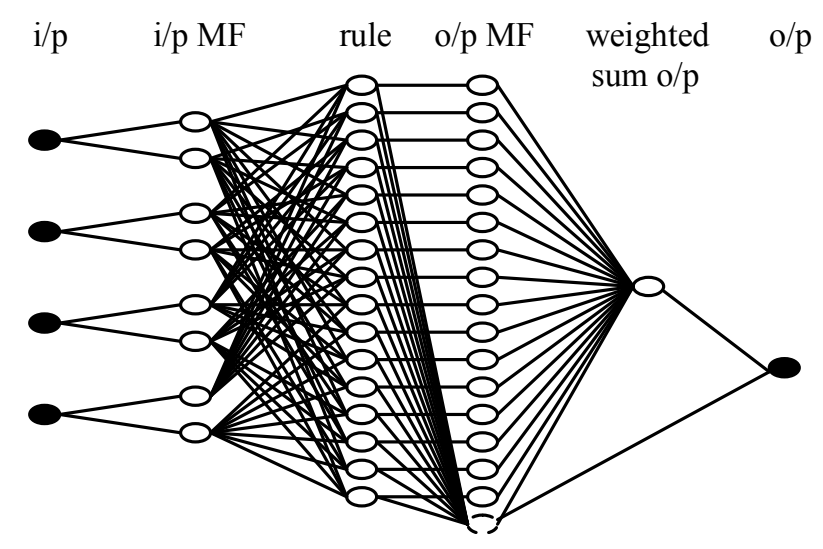

nodes - black: i/p - o/p, white: neurons, dashed: bias

Fig. 2. Neuro-fuzzy model architecture: layer 1 - inputs, layer 2 input membership functions, layer 3 - rules, layer 4 - output membership functions, layer 5 - weighted sum output, layer 6 - output. Black nodes indicate inputs and output of the neuro-fuzzy model whilst every white node indicates a single neuron. The white dashed node is a bias neuron.

node $i$ in this layer is an adaptive node using an output membership functions to compute the weighed output of the equivalent rule, according to the following node function: $O_{3, i}=\overline{w_{i}} f_{i}$ where $f_{i}=p_{i}+q_{i}+m_{i}+n_{i}+r_{i}$, and $\left\{p_{i}, q_{i}, m_{i}, n_{i}\right.$, $r_{i}$ \} are the consequent parameters (Konstantaras et al., 2004) of the network that specify the rules of the fuzzy inference system. In layer 5, the single node in this layer is a fixed node, which converts the weighted fuzzy outputs of all rules in the system into a single crisp output, as described by the following node function: $O_{4,1}=\sum_{i} \bar{w}_{i} f_{i}$. Finally, in layer 6 , the node describes the actual output of the neuro-fuzzy model for a given input data set.

A hybrid algorithm (Jang, 1993), a combination of the backpropagation algorithm (Jang et al., 1997) and the least squares estimator (Jang et al., 1997), maps the input data set to the output data set by adjusting accordingly the membership functions' and rules' parameters. In a similar way to feed-forward neural networks (Haykin, 1999), the neurofuzzy model generates an output signal during the forward pass, based on the initial set of membership functions. The consequent parameters are defined in the forward pass using the least squares method. Then the output is compared to the required output, and an error signal is fed back to the system to be used during the backward pass to readjust the premise parameters using the backpropagation algorithm. This process continues for a number of epochs until the error signal becomes sufficiently small (ideally zero) (Konstantaras et al., 2006a).
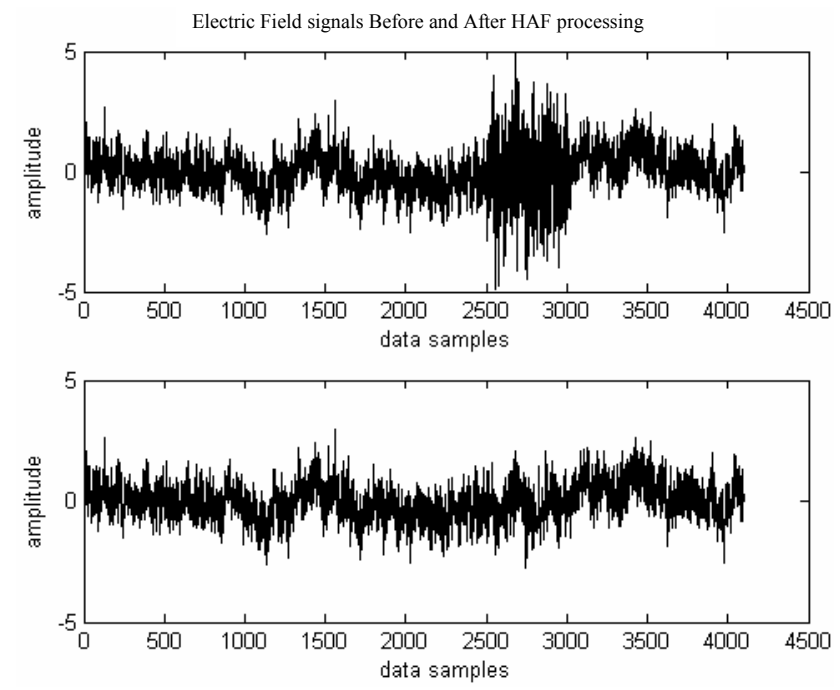

Fig. 3. Minimising the effect of magnetotelluric distortions upon the electric field: subplot 1 - electric field signal partially affected by severe transient fluctuations of magnetic origin (approximately between data samples 2500 and 3000), subplot 2 - the reconstructed electric field signal after being processed by the HAF method with the external distortion minimized (electric field signals have been down sampled by a factor of 4 with respect to their equivalent magnetic field signals for visualization purposes).

\section{Experimental results}

The neuro-fuzzy model was trained using a simulated magnetic field signal including significant distortions of similar effect to that of a magnetic storm, with known location and duration, at the input, and the simulated magnetic field signal alone as the required output. The performance of the HAF method and of the trained system was then tested using further unseen simulated electric field signals partially affected by noise external to the background signal approximately between data samples 2500 and 3000 (Fig 3, subplot 1) giving a signal to noise ratio of $4.19 \mathrm{~dB}$.

During the first stage of the HAF method the equivalent magnetic field signal is automatically derived from the original electric field signal, by reversing the magnetotelluric method. At this stage the magnetic field signal carries the same distortions, which had infected the original electric field signal, distinguished clearly approximately between data samples 10000 and 12000 (Fig. 4, subplot 1) exhibiting a signal to noise ratio of $6.41 \mathrm{~dB}$. Then, the neuro-fuzzy model, already trained as an adaptive filter, is activated upon the affected part of the data and minimizes the unwanted disturbances from the magnetic field signal. The external distortion on the resulting signal (Fig. 4, subplot 2) has been significantly reduced, leading to a signal to noise ratio of $21.07 \mathrm{~dB}$.

During the second stage of the HAF method the electric field signal is reconstructed (Fig. 3, subplot 2) based upon the processed magnetic field signal, which results in significant 
Table 1. This table demonstrates the signal to noise ratio (SNR) of another ten unseen simulated magnetic field signals (featuring the main characteristics of the Earth's magnetic field, i.e. chaotic time-series with a spectral density function that satisfies the relationship $S(\omega) \propto \omega^{-a}$ ) and equivalent electric field test-signals affected by severe transient fluctuations, before and after having been processed by the HAF method.

\begin{tabular}{|l|c|c|l|l|c|c|c|}
\hline $\begin{array}{l}\text { Magnetic } \\
\text { field signals }\end{array}$ & $\begin{array}{c}\text { SNR (dB) } \\
\text { before } \\
\text { neural } \\
\text { adaptation }\end{array}$ & $\begin{array}{c}\text { SNR (dB) } \\
\text { after } \\
\text { neural } \\
\text { adaptation }\end{array}$ & $\begin{array}{l}\text { Improvement } \\
\text { in SNR (dB) }\end{array}$ & $\begin{array}{l}\text { Equivalent } \\
\text { electric field } \\
\text { signals }\end{array}$ & $\begin{array}{c}\text { SNR (dB) } \\
\text { before } \\
\text { neural } \\
\text { adaptation }\end{array}$ & $\begin{array}{c}\text { SNR (dB) } \\
\text { after } \\
\text { neural } \\
\text { adaptation }\end{array}$ & $\begin{array}{l}\text { Improvement } \\
\text { in SNR (dB) }\end{array}$ \\
\hline test signal 1 & 6.41 & 21.07 & 14.66 & test signal 1 & 4.19 & 19.74 & 15.55 \\
\hline test signa1 2 & 5.91 & 20.87 & 14.96 & test signal 2 & 3.62 & 18.29 & 14.67 \\
\hline test signa1 3 & 6.37 & 20.61 & 14.24 & test signal 3 & 4.25 & 19.03 & 14.78 \\
\hline test signa1 4 & 8.01 & 21.80 & 13.79 & test signal 4 & 5.39 & 19.98 & 14.59 \\
\hline test signal 5 & 9.67 & 20.37 & 10.70 & test signal 5 & 5.77 & 20.56 & 14.79 \\
\hline test signa16 & 10.28 & 20.55 & 10.27 & test signal 6 & 6.02 & 19.78 & 13.76 \\
\hline test signa17 & 9.51 & 20.72 & 11.21 & test signal 7 & 5.91 & 19.75 & 13.84 \\
\hline test signa18 & 8.70 & 19.73 & 11.03 & test signa1 & 5.64 & 19.67 & 14.03 \\
\hline test signa19 & 8.33 & 19.99 & 11.66 & test signal9 & 4.98 & 18.88 & 13.90 \\
\hline test signal 10 & 8.09 & 20.29 & 12.20 & test signal 10 & 5.14 & 19.31 & 14.17 \\
\hline
\end{tabular}
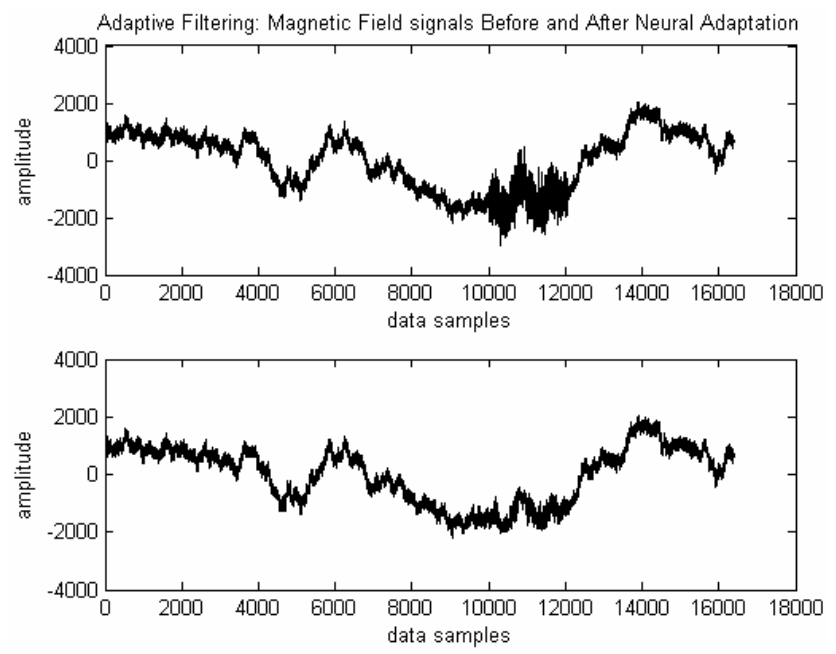

Fig. 4. Minimisation of severe transient fluctuations upon magnetic field time-series (approximately between data samples 10000 and $12000)$ using adaptive filtering: subplot 1 - magnetic field signal before neural adaptation, subplot 2 - magnetic field signal after neural adaptation $(\mathrm{fs}=1 \mathrm{~Hz})$.

reductions on the noise level of the former in comparison to the original electric field signal. The signal to noise ratio of the electric field signal after neural adaptation equals $19.74 \mathrm{~dB}$, which is in balance with the noise level of random noise appearing on the recorded data estimated at approximately 20 dB (Makris, 1997; d'Erceville and Kunetz; Groom, 1988).

Table 1 outlines results obtained using a further ten unseen magnetic and electric field test-signals, reinforcing earlier observations on the performance of the HAF method. In every case there is a significant improvement in signal to noise ratio when the HAF method is used, with the average improvement for the ten magnetic field test signals measured at $12.48 \mathrm{~dB}$ and the average improvement for the ten electric field test signals measured at $14.41 \mathrm{~dB}$.

\section{Conclusions}

These results clearly demonstrate the ability of adaptive networks to process dynamic signals and the effectiveness of neuro-fuzzy models as adaptive filters. The development of the HAF method incorporating neuro-fuzzy technology provides an effective mechanism that minimizes any significant disturbances of external origin to the background signal superimposed on electrotelluric field recordings. Thus, the method is valid not only for magnetic storms, but it can be applied to remove man-made noises and other spurious effects. Providing that the impedance tensor of the subsoil at the observation site is known, there are no limitations regarding the site's location. Furthermore, noise minimisation from electric field recordings is achieved by operating on the equivalent magnetic field signal thereby preserving any "external" information added on the electric field, such as EEP signals.

Acknowledgements. This work was supported from the project Archimedes I, sub-project No 2.2.15 entitled: Multiparametric tempo-spatial estimation of the seismic hazard at the front of the Hellenic arc. Application to the protection of the old town and the Venetian port of Chania in the frame of the Operational Programme for Education and Initial Vocational Training (O.P. "Education"), co-financed from European Social Fund of European Union and national resources of Greece.

Edited by: P. F. Biagi

Reviewed by: V. Lapenna and two other referees 


\section{References}

Cagniard, L.: Basic theory of the magneto-telluric method of geophysical prospecting, Geophysics, 18, 605-635, 1953.

d'Erceville, I. and Kunetz, G.: Some observations regarding naturally occurring electromagnetic fields in applied geophysics, Geophysics, 27, pp. 651, 1962.

Groom, W. R.: The effects of inhomogeneities on Magnetotellurics, Ph.D. Thesis, University of Toronto, Canada, 1988.

Hadjioannou, D. and Vallianatos, F.: Subtraction of the telluric inductive component from VAN measurements, Tectonophysics, 224, 113-124, 1993.

Haykin, S.: Neural Networks, 2nd Edition, Prentice Hall, 1999.

Jang, J.: ANFIS: Adaptive Network-based Fuzzy Inference Systems, IEEE Transactions on Systems, Man, and Cybernetics, 23(03), 665-685, 1993.

Jang, J., Sun, T., and Mizutany, E.: Neuro-fuzzy and soft computing, Prentice Hall, Upper Saddle River, NJ, 1997.

Kaufman, A. A. and Keller, G. V.: The magnetotelluric sounding method, Elsevier, 15, pp. 583, 1981.

Konstantaras, A., Varley, M. R., Vallianatos, F., Collins, G., and Holifield, P.: Recognition of electric earthquake precursors using neuro-fuzzy methods: methodology and simulation results, Proc. IASTED Int. Conf. On Signal Processing, Pattern Recognition and Applications (SPPRA 2002), Crete, Greece, 303-308, 2002.

Konstantaras, A., Varley, M. R., Vallianatos, F., Collins, G., and Holifield, P.: A neuro-fuzzy approach to the reliable recognition of electric earthquake precursors, Nat. Hazards Earth Syst. Sci., 4, 641-646, 2004,

http://www.nat-hazards-earth-syst-sci.net/4/641/2004/.
Konstantaras, A., Varley, M. R., Vallianatos, F., Collins, G., and Holifield, P.: Neuro-fuzzy prediction-based adaptive filtering applied to severely distorted magnetic field recordings, IEEE Geosci. Remote Sens. Lett., 3(4), 439-441, $2006 a$.

Konstantaras, A., Varley, M. R., Vallianatos, F., Makris, J. P., Collins, G., and Holifield, P.: Detection of weak seismo-electric signals upon the recordings of the electrotelluric field by means of neuro-fuzzy technology, IEEE Geosci. Remote Sens. Lett., in press, 2006b.

Makris, J. P.: Electromagnetic study of the geoelectric structure of areas sensitive in the detection of Electric Earthquake Precursors, Ph.D. Thesis, Kapodistrian University of Athens, Greece, 1997.

Rovithakis, G. and Vallianatos, F.: A neural network approach to the identification of electric earthquake precursors, Phys. Chem. Earth, 25(3), 315-319, 2000.

Tzanis, A. and Vallianatos, F.: A critical review of electric earthquake precursors, Annali di Geofisica, 44(2), 429-460, 2001.

Vallianatos, F., Lapenna, V., Troyan, V., Smirnova, N., Kopytenko, Y., Korepanov, V., and Matiashvili, T.: Study of the ULF electromagnetic phenomena related to earthquakes: Strategy of the SUPRE project, Seismo Electromagnetics LithosphereAtmosphere-Ionosphere Coupling, TERRAPUB, Tokyo, Japan, 437-442, 2002.

Varotsos, P. and Alexopoulos, K.: Physical Properties of the variations of the electric field of the Earth preceding earthquakes, I, Tectonophysics, 110, 73-98, 1984. 